\title{
Editorial
}

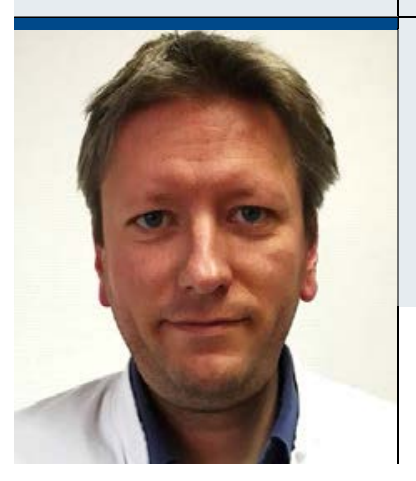

"Sexuell übertragbare Erkrankungen zu behandeln,

gehört unbestritten zur Kernkompetenz der Urologen."

\section{Offenheit in der Urologie und Gesellschaft gefordert}

D ie Urologie mag zwar als Medizin des alten Mannes wahrgenommen werden, doch beweist die tägliche Praxis, dass vor allem junge, an sich gesunde Menschen mehr und mehr den urologischen Facharzt aufsuchen - meist aufgrund von Beschwerden durch eine sexuell übertragbare Infektion.

In der Gesellschaft werden die sogenannten Geschlechtskrankheiten jedoch weiterhin tabuisiert. $\mathrm{Zu}$ groß ist die Angst der Infizierten vor Diskriminierung und sozialer Isolation. Zudem besteht für einige Erkrankungen wie beispielsweise die Gonorrhö keine Meldepflicht mehr. Bundesweite Screeningprogramme, wie man sie für junge Frauen vorsieht, werden für Risikogruppen wie Sexualarbeiter oder Männer, die Sex mit Männern haben (MSM), leider trotz Empfehlung medizinischer Fachgesellschaften nicht unterstützt.

\section{Deutsche Leitlinie im Vergleich schärfer und konsequenter}

Verfügbare epidemiologische Daten und zunehmende Resistenzraten der ursächlichen Erreger unterstreichen jedoch nicht nur die Aktualität in Deutschland. Sie verdeutlichen auch eine bedenkliche Entwicklung, die einen offenen Umgang mit der Thematik fordert. Um dieser Problematik zu begegnen, wurde unlängst in den deutschen Leitlinienempfehlungen reagiert. Wie am Beispiel der gonorrhoischen Urethritis ersichtlich, wird in Deutschland mittlerweile eine duale hochdosierte antimikrobielle Therapie als Standardtherapie empfohlen verbunden mit dem primären Ziel des Erregernachweises für die Urethritisdiagnostik. Aufgrund der
Resistenzproblematik bei Gonokokken wird im Vergleich zu internationalen Leitlinien ein konsequenterer und schärferer Weg verfolgt.

\section{Zusammenschluss bewirkt Therapieerfolg}

Sexuell übertragbare Erkrankungen zu behandeln, gehört unbestritten zur Kernkompetenz der Urologen. Im Sinne von Aufklärung, Prävention und adäquater Therapie ist es notwendig, die aktuellen Empfehlungen umzusetzen. Um die Krankheit zu managen, ist ein interdisziplinärer Ansatz der Urologie mit anderen Disziplinen wie der Gynäkologie, Dermatologie und inneren Medizin sinnvoll.

Die Autoren der aktuellen Ausgabe der URONEWS informieren über relevante und häufige sexuell übertragbare Infektionen. Dabei spannen sie einen weiten Bogen von der Gonorrhö über die Syphilis, die weiterhin zunehmende Inzidenzen aufweist, bis hin zu differentialdiagnostisch bedeutenden Erkrankungen wie das Lymphogranuloma venereum.

Ein besonderer Dank gilt ausdrücklich Herrn Giuseppe Magistro, der maßgeblich an der Themengestaltung und Ausarbeitung der Beiträge beteiligt war.

Die Autoren wünschen den Leserinnen und Lesern eine interessante und informative Lektüre der Beiträge.

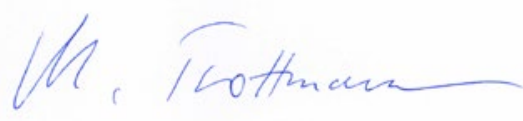

